\title{
Application of the multiaxial perfectly matched layer (M-PML) to near-surface seismic modeling with Rayleigh waves
}

\author{
Chong Zeng ${ }^{1}$, Jianghai Xia ${ }^{1}$, Richard D. Miller ${ }^{1}$, and Georgios P. Tsoflias ${ }^{2}$
}

\begin{abstract}
Perfectly matched layer (PML) absorbing boundaries are widely used to suppress spurious edge reflections in seismic modeling. When modeling Rayleigh waves with the existence of the free surface, the classical PML algorithm becomes unstable when the Poisson's ratio of the medium is high. Numerical errors can accumulate exponentially and terminate the simulation due to computational overflows. Numerical tests show that the divergence speed of the classical PML has a nonlinear relationship with the Poisson's ratio. Generally, the higher the Poisson's ratio, the faster the classical PML diverges. The multiaxial PML (M-PML) attenuates the waves in PMLs using different damping profiles that are proportional to each other in orthogonal directions. The proportion coefficients of the damping profiles usually vary with the specific model settings. If they are set appropriately, the
\end{abstract}

M-PML algorithm is stable for high Poisson's ratio earth models. Through numerical tests of 40 models with Poisson's ratios that varied from 0.10 to 0.49 , we found that a constant proportion coefficient of 1.0 for the $\mathrm{x}$ - and $\mathrm{z}$-directional damping profiles is sufficient to stabilize the M-PML for all 2D isotropic elastic cases. Wavefield simulations indicate that the instability of the classical PML is strongly related to the wave phenomena near the free surface. When applying the multiaxial technique only in the corners of the PML near the free surface, the original M-PML technique can be simplified without losing its stability. The simplified M-PML works efficiently for homogeneous and heterogeneous earth models with high Poisson's ratios. The analysis in this paper is based on 2D finite difference modeling in the time domain that can easily be extended into the $3 \mathrm{D}$ domain with other numerical methods.

\section{INTRODUCTION}

With the increasing demands on environmental and engineering studies, modeling seismic wave propagation in the near surface is essential and fundamental. The relationship between Rayleigh-wave phase velocity and frequency has been widely utilized to estimate the S-wave velocities in shallow layers (Nazarian and Stokoe, 1984; Xia et al., 1999, 2003, 2006; Calderón-Macías and Luke, 2007; Socco et al., 2010). Hence, generating synthetic records containing accurate Rayleigh-wave information is a primary objective of any near-surface seismic modeling task. High Poisson's ratio earth models are often employed in the near-surface studies. Many near-surface materials are unlithified and have much higher Poisson's ratios than the sedimentary rocks. For example, Xia et al. (2002) showed that the materials of upper $7 \mathrm{~m}$ at a mining site in Wyoming, U.S.A., have the Poisson's ratio of about 0.48. They also reported that the unconsolidated sediments of the Fraser River Delta near Vancouver, Canada, have the Poisson's ratio of about 0.49 , which is close to the maximum theoretical Poisson's ratio (0.5). Modeling Rayleigh waves in high Poisson's ratio earth models is critical to many near-surface geophysical studies.

Rayleigh waves can be simulated through numerical methods, such as finite-difference (FD) method, by applying appropriate free-surface boundary conditions (e.g., Mittet, 2002; Xu et al., 2007). Absorbing boundary conditions are usually employed to suppress spurious reflections from the truncated edges of a finite-sized discrete earth model. Cerjan et al. (1985) introduced a sponge-layer absorbing boundary condition for discrete elastic wave equations. The absorbing effectiveness of this method

\footnotetext{
Manuscript received by the Editor 14 October 2010; revised manuscript received 11 December 2010; published online 28 April 2011.

${ }^{1}$ The University of Kansas, Kansas Geological Survey, Lawrence, Kansas, U.S.A. E-mail: czeng@kgs.ku.edu; jxia@kgs.ku.edu; rmiller@kgs.ku.edu.

${ }^{2}$ The University of Kansas, Department of Geology, Lawrence, Kansas, U.S.A. E-mail: tsoflias@ku.edu. (C) 2011 Society of Exploration Geophysicists. All rights reserved.
} 
depends to a large extent on the distance that the waves propagate in the transition zone. The damping strip has to be wide enough to yield satisfactory attenuation results, thereby greatly increasing the computational expense. Bérenger (1994) developed an improved absorbing boundary condition for attenuating electromagnetic waves. This technique utilizes an absorbing layer called the perfectly matched layer (PML) to generate a nonreflecting interface between the artificial boundary and the free medium. Subsequently, the PML method was successfully introduced to elastic wavefield studies (Chew and Liu, 1996; Collino and Tsogka, 2001). It is now the most widely used technique for solving the spurious reflection problem in seismic modeling.

The PML method is based on a nonphysical modification to the wave equation inside the absorbing strip so that the theoretical reflection coefficient at the strip-model interface is zero. It allows reduction in the width of the transition zone to nearly $25 \%$ of the classical sponge absorbing methods (Carcione et al., 2002). Festa and Nielsen (2003) show that the PML method is efficient in the presence of strong Rayleigh waves.

For near-surface seismic modeling, Rayleigh waves dominate the P-SV (the coupling of compressional waves and the vertical components of shear waves) wavefield (e.g., Xia et al., 2002; Saenger and Bohlen, 2004). Compared to conventional seismic modeling that focuses on P-waves, a higher spatial sample density of grid points per wavelength (ppw) is required to avoid the numerical dispersion of Rayleigh waves during the model discretization procedure (Mittet, 2002). The increased spatial sample density causes an increase in the number of model grids over those in conventional seismic modeling, costing more computer memory and central processing unit (CPU) time. Employing the PML technique can tremendously reduce the cost of computation. However, in many cases the performance of classical PML absorption (refer to the implementation of Collino and Tsogka [2001]) does not meet the expectations of near-surface seismic modeling. For a fine grid near-surface earth model, the time step size during the FD modeling is usually less than $0.1 \mathrm{~ms}$ so that the Courant-Friedrichs-Lewy (CFL) condition is satisfied to ensure the stability of the modeling algorithm. In this case, the number of time marching loops is greater than 10,000 to generate a synthetic record of 1-s time length. The accumulative errors can be significant, which makes the PML algorithm diverge and causes a computational instability problem during the modeling. Komatitsch and Martin (2007) introduced a convolutional PML (C-PML) technique as a general representation of the classical PML method to improve the absorbing effectiveness at grazing incidence. However, the instability problem still appears in simulations performed for long time duration.

Physical properties of the medium can cause the PML algorithm to be intrinsically unstable. For some anisotropic media reported by Bécache et al. (2003), the classical PML and C-PML techniques suffer from the instability problem (Komatitsch and Martin, 2007). For a near-surface medium that has a high Poisson's ratio $(>0.4)$, we also found that neither the classical PML nor the C-PML is stable even for a simple isotropic elastic case with the existence of the free surface. The application of the classical PML to modeling Rayleigh waves in near-surface materials is challenging due to the instability of PML in high Poisson's ratio earth models.

Classical PML and C-PML techniques can be considered uniaxial PML methods. Waves in uniaxial PMLs are attenuated in only one direction using a unique damping factor. Mesa-Fajardo and Papageorgiou (2008) conducted a comprehensive mathematical analysis on the stability of the classical PML method. They further developed a multiaxial PML (M-PML) method through eigenvalue sensitivity analysis that improved on the stability of the original method (PML). The M-PML is based on a more general coordinate stretching version of the classical split-field PML, in which the waves are attenuated in all directions with different damping factors (hence the name "multiaxial"). A stable M-PML algorithm can be constructed by tuning the proportion coefficients of the damping factors according to the settings of a specific model. This approach was successfully applied to modeling seismic waves in an orthotropic medium (Mesa-Fajardo and Papageorgiou, 2008), where the classical PML is intrinsically unstable.

In this paper, we present the instability problem of the classical uniaxial PML commonly observed in media with different Poisson's ratios. In the numerical tests a critical Poisson's ratio can be estimated as the lowest value of the ratio when the PML becomes unstable. Then we test the stability of the M-PML method using the same models with various Poisson's ratios. We also show that the multiaxial technique is only necessary for the model grids that are near the free surface. Based on this observation, we slightly simplified the original M-PML by setting the absorbing zones only near the free surface to be multiaxial. Finally, we demonstrate the stability of this simplified M-PML through its application to a layered near-surface earth model. The analysis presented here is based on time domain, 2D finite-difference modeling. However, the simplification of the M-PML approach can be extended in a straightforward fashion to the $3 \mathrm{D}$ case using other numerical methods such as finiteelement, pseudospectral, and spectral-element methods.

\section{MODELING OF RAYLEIGH WAVES WITH CLASSICAL PML}

The vector wave equation in an isotropic medium (Aki and Richards, 2002) is

$$
\rho \ddot{\mathbf{u}}=\mathbf{f}+(\lambda+2 \mu) \nabla(\nabla \cdot \mathbf{u})-\mu \nabla \times(\nabla \times \mathbf{u}),
$$

where $\rho$ is the mass density, $\mathbf{u}$ is the displacement vector, $\ddot{\mathbf{u}}$ is the second derivative of the displacement vector with respect to time, $\mathbf{f}$ is the body force vector, and $\lambda$ and $\mu$ are the Lamé coefficients. A first-order velocity-stress form of the wave equation can be formulated by differentiating the displacement field with respect to time. In a $2 \mathrm{D}$ vertical plane, it can be written as the following set of equations with the stress-strain relations (Virieux, 1986):

$$
\begin{aligned}
& \frac{\partial v_{x}}{\partial t}=b\left(\frac{\partial \tau_{x x}}{\partial x}+\frac{\partial \tau_{x z}}{\partial z}\right) \\
& \frac{\partial v_{z}}{\partial t}=b\left(\frac{\partial \tau_{x z}}{\partial x}+\frac{\partial \tau_{z z}}{\partial z}\right) \\
& \frac{\partial \tau_{x x}}{\partial t}=(\lambda+2 \mu) \frac{\partial v_{x}}{\partial x}+\lambda \frac{\partial v_{z}}{\partial z} \\
& \frac{\partial \tau_{z z}}{\partial t}=(\lambda+2 \mu) \frac{\partial v_{z}}{\partial z}+\lambda \frac{\partial v_{x}}{\partial x} \\
& \frac{\partial \tau_{x z}}{\partial t}=\mu\left(\frac{\partial v_{x}}{\partial z}+\frac{\partial v_{z}}{\partial x}\right)
\end{aligned}
$$


where $\left(v_{x}, v_{z}\right)$ is the particle velocity vector, $b(x, z)$ is the buoyancy (the reciprocal of mass density), $\left(\tau_{x x}, \tau_{z z}, \tau_{x z}\right)$ is the stress vector, and $t$ is the time variable. The initial condition is that at time $t=0$, all the velocities and stresses are set to zero throughout the model. A discretization procedure can be performed using the well-known Madariaga-Virieux staggered grid scheme (Madariaga, 1976; Virieux, 1986) to ensure the stability in a heterogeneous medium with large variations of Poisson's ratio. We use the staggered-grid form presented by Graves (1996) with fourth-order accurate space and second-order accurate time (Levander, 1988) during implementation of the FD modeling. For the grids located on the free surface, parameters are updated through a fourth-order FD scheme developed by Kristek et al. (2002). For the internal model grids, a parameter averaging technique proposed by Moczo et al. (2002) is used to improve model stability. By applying a source excitation to the velocity components, particle velocities can be calculated through a time marching scheme. Rayleigh waves can be modeled with the simulation of P-SV wave propagation.

The PMLs are attached by surrounding the physical domain of the model with three transition strips on the left, right, and bottom sides, respectively (Figure 1). They can be interpreted by the continuation of the physical model domain using a coordinate stretching theory (Chew and Liu, 1996). By constructing a PML differential operator and decomposing the stresses and velocities in orthogonal directions, the $2 \mathrm{D}$ wave equation can be rewritten as (Collino and Tsogka, 2001)

$$
\begin{gathered}
v_{x}=v_{x}^{x}+v_{x}^{z} ; \quad v_{z}=v_{z}^{x}+v_{z}^{z} \\
\left(\partial_{t}+d_{x}\right) v_{x}^{x}=b \frac{\partial \tau_{x x}}{\partial x} \\
\left(\partial_{t}+d_{z}\right) v_{x}^{z}=b \frac{\partial \tau_{x z}}{\partial z} \\
\left(\partial_{t}+d_{x}\right) v_{z}^{x}=b \frac{\partial \tau_{x z}}{\partial x} \\
\left(\partial_{t}+d_{z}\right) v_{z}^{z}=b \frac{\partial \tau_{z z}}{\partial z},
\end{gathered}
$$

with the stress-strain relations:

$$
\begin{aligned}
\tau_{x x}=\tau_{x x}^{x}+\tau_{x x}^{z} ; \tau_{z z} & =\tau_{z z}^{x}+\tau_{z z}^{z} ; \tau_{x z}=\tau_{x z}^{x}+\tau_{x z}^{z} \\
\left(\partial_{t}+d_{x}\right) \tau_{x x}^{x} & =(\lambda+2 \mu) \frac{\partial v_{x}}{\partial x} \\
\left(\partial_{t}+d_{z}\right) \tau_{x x}^{z} & =\lambda \frac{\partial v_{z}}{\partial z} \\
\left(\partial_{t}+d_{x}\right) \tau_{z z}^{x} & =\lambda \frac{\partial v_{x}}{\partial x} \\
\left(\partial_{t}+d_{z}\right) \tau_{z z}^{z} & =(\lambda+2 \mu) \frac{\partial v_{z}}{\partial z} \\
\left(\partial_{t}+d_{x}\right) \tau_{x z}^{x} & =\mu \frac{\partial v_{z}}{\partial x} \\
\left(\partial_{t}+d_{z}\right) \tau_{x z}^{z} & =\mu \frac{\partial v_{x}}{\partial z}
\end{aligned}
$$

where $d_{x}$ and $d_{z}$ are the PML damping profiles along $x$ (horizontal) and $z$ (vertical) directions, respectively. The superscript $x$ and $z$ represent the split PML components in $x$ - and $z$-directions, respectively. This is a nonphysical decomposition to the velocity and stress vectors so as to accommodate the attenuation algorithm of PML. Within the physical model domain, $d_{x}$ and $d_{z}$ are zero so that equations 3 and 4 degrade to equation 2 . If the damping profiles in the PMLs are well designed, waves can be attenuated with no significant spurious reflections coming from either the truncated model edges or the interfaces of the PMLs and the physical model domain.

In the classical PML, waves are only attenuated in one direction (uniaxial). For example, within the left and right PMLs shown in Figure 1, only the damping factor along the $x$-direction is nonzero. That is

$$
d_{x}=d_{x}(x), d_{z}=0 .
$$

Similarly, within the bottom PML, only the damping profile along the $z$ direction takes effect:

$$
d_{x}=0, d_{z}=d_{z}(z) .
$$

For the bottom-left and bottom-right corners, the $x$ and $z$ damping profiles naturally superpose together, making the wave decay in all the directions. However, for the upper-left and upper-right corners, the PMLs should attenuate the waves in only the $x$-direction. Otherwise strong spurious reflections of Rayleigh waves will occur at the interface between the PML and the physical domain.

The classical PML method works efficiently when the Poisson's ratio of a medium is low. Figure 2 displays wavefield simulation snapshots (particle velocities in the $z$-direction) when a point source vertically excites the free surface of a Poisson's solid model (the Poisson's ratio $\sigma=0.25$ ). The source wavelet is the first derivative of the Gaussian function defined as

$$
w(t)=2 \pi f\left(t-t_{0}\right) e^{-\pi^{2} f^{2}\left(t-t_{0}\right)^{2}},
$$

where $f$ is the dominant frequency, and $t_{0}$ is the time zero delay. Because the effectiveness of PML absorption is independent of the source frequency according to its developing procedure (Bérenger, 1994; Collino and Tsogka, 2001), we use $f=50 \mathrm{~Hz}$ and $t_{0}=24 \mathrm{~ms}$ for all the examples provided in this paper unless otherwise stated. For the models in Figure 2, the minimum PML

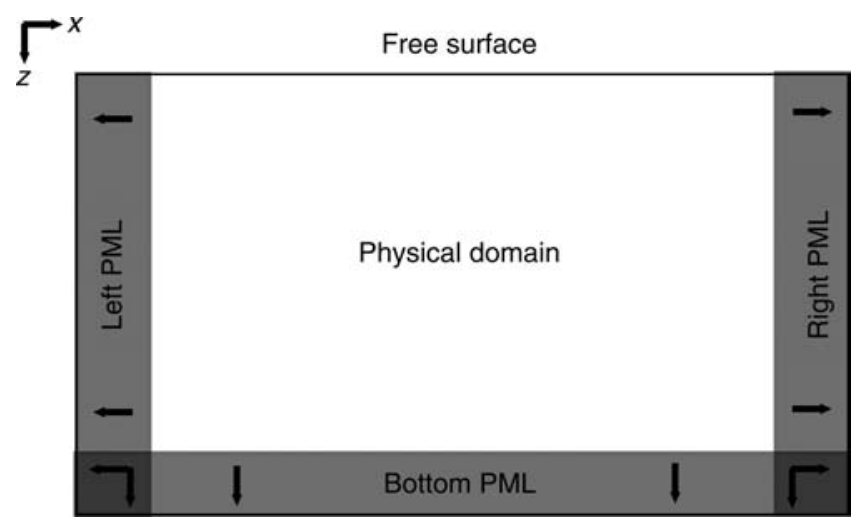

Figure 1. A sketch of the PML absorbing layers in a 2D domain. The physical model domain is surrounded by three PMLs. The arrows represent the attenuation direction of the waves inside PMLs. For the lower-left and lower-right corners of the PMLs, the damping profiles are superposed together naturally. For the implementation of uniaxial PML technique, the overlapping in the corner has only two components. While in the M-PML technique, it is implemented by the superposition of four damping profiles. 
thickness is only $1 / 4$ of the dominant wavelength of the P-waves. The body waves and Rayleigh waves decay in the PMLs with no significant spurious reflections.

\section{STABILITY TESTS OF CLASSICAL PML FOR NEAR-SURFACE EARTH MODELS}

The complexity of shallow earth materials can make the application of classical PML challenging. A common factor that yields instability is a high Poisson's ratio in the near surface medium. Many unlithified materials in the near surface have Poisson's ratios greater than 0.4. Some near-surface materials such as saturated sand can even have a Poisson's ratio close to 0.5. In those media, the near-surface wavefield is complicated due to the intricate interaction of various waves with the free surface. A high Poisson's ratio near the free surface introduces difficulties to the absorption of PMLs for near-surface earth models. Numerical errors can be accumulated to significant values in the PMLs after discretization. The classical uniaxial PML
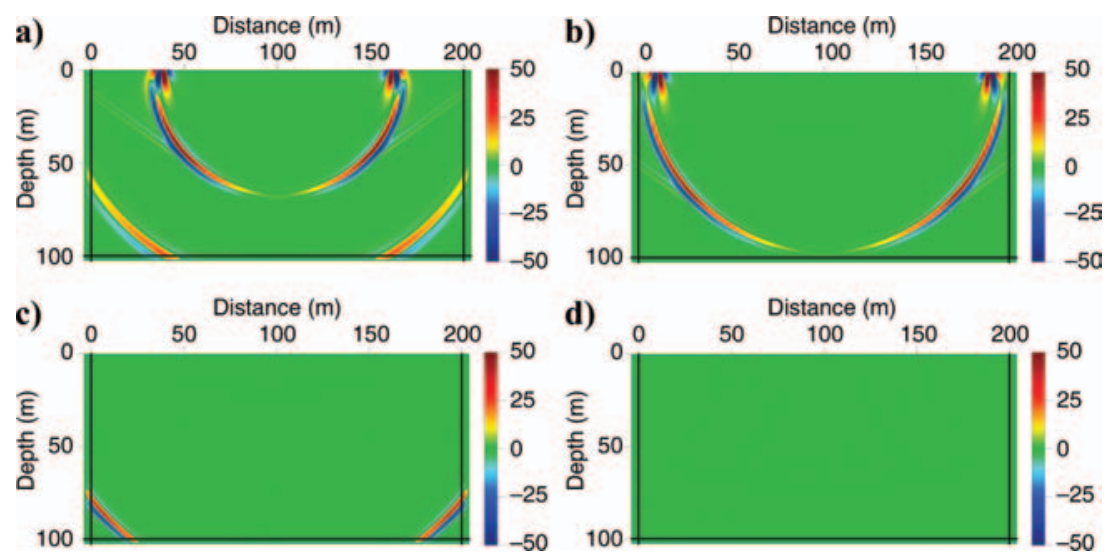

Figure 2. (a) Snapshot of the vertical particle velocity for a Poisson's solid homogeneous half-space earth model with the classical PML at time instant $t=250 \mathrm{~ms}$. Solid lines are the interfaces between the PML and the physical model domain. The source is located at $(x, z)=(100 \mathrm{~m}, 0 \mathrm{~m})$. The $\mathrm{P}$-wave velocity, S-wave velocity, and mass density in the model are $520 \mathrm{~m} / \mathrm{s}, 300 \mathrm{~m} / \mathrm{s}$, and $1.5 \times 10^{3} \mathrm{~kg} / \mathrm{m}^{3}$, respectively. The width of the left and right PMLs are $4 \mathrm{~m}$. The width of the bottom PML is $2.6 \mathrm{~m}$. The body wave and surface wave are attenuated efficiently without significant spurious reflections. (b) Snapshot of the vertical particle velocity for a Poisson's solid homogeneous half-space earth model with the classical PML at time instant $t=350 \mathrm{~ms}$. Solid lines are the interfaces between the PMLs and the physical model domain. The source is located at $(x, z)=(100 \mathrm{~m}, 0 \mathrm{~m})$. The $\mathrm{P}$-wave velocity, $\mathrm{S}$-wave velocity, and mass density in the model are $520 \mathrm{~m} / \mathrm{s}, 300 \mathrm{~m} / \mathrm{s}$, and $1.5 \times 10^{3} \mathrm{~kg} / \mathrm{m}^{3}$, respectively. The width of the left and right PMLs are $4 \mathrm{~m}$. The width of the bottom PML is $2.6 \mathrm{~m}$. The body wave and surface wave are attenuated efficiently without significant spurious reflections. (c) Snapshot of the vertical particle velocity for a Poisson's solid homogeneous half-space earth model with the classical PML at time instant $t=450 \mathrm{~ms}$. Solid lines are the interfaces between the PMLs and the physical model domain. The source is located at $(x, z)=(100 \mathrm{~m}, 0 \mathrm{~m})$. The $\mathrm{P}$-wave velocity, S-wave velocity, and mass density in the model are $520 \mathrm{~m} / \mathrm{s}, 300 \mathrm{~m} / \mathrm{s}$, and $1.5 \times 10^{3} \mathrm{~kg} / \mathrm{m}^{3}$, respectively. The width of the left and right PMLs are $4 \mathrm{~m}$. The width of the bottom PML is $2.6 \mathrm{~m}$. The body wave and surface wave are attenuated efficiently without significant spurious reflections. (d) Snapshot of the vertical particle velocity for a Poisson's solid homogeneous half-space earth model with the classical PML at time $t=550 \mathrm{~ms}$. Solid lines are the interfaces between the PMLs and the physical model domain. The source is located at $(x, z)=(100 \mathrm{~m}, 0 \mathrm{~m})$. The P-wave velocity, S-wave velocity, and mass density in the model are $520 \mathrm{~m} / \mathrm{s}, 300 \mathrm{~m} / \mathrm{s}$, and $1.5 \times 10^{3} \mathrm{~kg} / \mathrm{m}^{3}$, respectively. The width of the left and right PMLs are $4 \mathrm{~m}$. The width of the bottom PML is $2.6 \mathrm{~m}$. The body wave and surface wave are attenuated efficiently without significant spurious reflections. algorithm is unstable during the modeling even for a simple

Figure 3 shows the wavefield snapshots for a homogeneous velocity $\left(v_{s}\right)$ in the model are $520 \mathrm{~m} / \mathrm{s}$ and $102 \mathrm{~m} / \mathrm{s}$, respecFD implementation, the model is uniformly discretized into (pells so that the grid sample density is sufficien the FD algorithm is numerically stable. The PML thickness in and $z$-directions are $10 \mathrm{~m}$, which is about a dominant wavelength of the P-waves.

On the snapshot at $t=115 \mathrm{~ms}$ (Figure $3 \mathrm{a}$ and e), the body waves enter the bottom PML with no significant spurious reflec( larly, the waves are attenuated immediately after they enter the PMLs at $t=132 \mathrm{~ms}$ (Figure $3 \mathrm{~b}$ and $\mathrm{f}$ ). However, when the wavefronts approach the left and right external model edges, the absorption in the left and right PMLs does not meet expectations. Small numerical errors appear at the upper-left and upper-right corners of the PMLs on the $t=139 \mathrm{~ms}$ snapshot (Figure $3 \mathrm{c}$ and g). With time marching, the amplitudes of particle velocities near the model edges increase exponentially (e.g., the snapshot in Figure $3 d$ and h). The error propagates with spurious reflections from the model edges and accumulates abruptly in the PML. This indicates the PML algorithm loses its stability for this model. The computation is finally terminated after about 2980 time marching loops due to the numerical overflow.

To test if the instability is caused by the model discretization, we change the model parameter configuration by reducing the grid spacing of the model to $0.025 \times 0.025 \mathrm{~m}$ and run the simulation again. The physical thickness of the PML is still $10 \mathrm{~m}$. In this case, the spatial grid sample density in the PML is 16 times of that in the previous simulation. The time step size is also reduced to $0.0125 \mathrm{~ms}$. This is a finer discretization than the previous configuration. The computation is terminated after about 10,720 time marching loops, which is much greater than the number in the previous test. Comprehensive tests show that the program survives with different loop times with various model settings (e.g., grid spacing, time step size, etc.). This confirms that the instability of the PML is related to the discretization of the model and mainly controlled by the accumulated numerical errors.

Although the mathematical analysis on the stability of PML methods is presented by MezaFajardo and Papageorgiou (2008), there is no conclusive criterion related to the model's physical parameters to indicate under what conditions the classical PML is unstable. However, 
by comparing the unstable modeling results in Figure 3 with those in Figure 2 where the classical PML works well, it suggests that the stability of the classical PML is closely related to the values of Poisson's ratios.

Numerical testing is a convenient way to provide an estimation how the Poisson's ratio affects the stability of the classical PML. Here we test 40 models with Poisson's ratios varying from 0.10 to 0.49 . The detailed physical parameters of the models are listed in Table 1. All the models are constructed with a $50 \times 50 \mathrm{~m}$ physical domain surrounded by three $10-\mathrm{m}$-wide PMLs. The P-wave velocity and mass density remain constants in all the 40 models as $520 \mathrm{~m} / \mathrm{s}$ and $1.5 \times 10^{3} \mathrm{~kg} / \mathrm{m}^{3}$, respectively. The point source is horizontally centered on the free surface. The grid spacing in the $x$ - and $z$-directions is $0.1 \mathrm{~m}$. The simulation time is $2 \mathrm{~s}$ with a time marching step size of 0.05 ms. The maximum number of time marching loops is 40,000 , which is large enough to allow the error to accumulate to a significant value if the PML algorithm is unstable.

Because all the test models are homogenous, the kinetic energy $E=\frac{1}{2} m v^{2}$ for each particle of the model can be compared directly using the amplitude of the velocities. For the source wavelet defined in equation 7 , the maximum velocity value of the source particle is less than $1.0 \mathrm{~m} / \mathrm{s}$. Consequently, in accordance with the laws of energy conservation none of the particle velocity amplitudes in the model can be greater than 1.0 $\mathrm{m} / \mathrm{s}$. However, if the PML algorithm is divergent, this threshold can be exceeded due to the rapid accumulation of numerical errors. So the PML algorithm would be considered unstable once the velocity threshold is broken during the modeling time marching procedure. The modeling program is designed to terminate immediately in this situation. Table 1 lists the maximum number of time marching steps for each model. When the number of time marching steps is 40,000 the modeling was completed without an abnormal termination. In other words, the PML algorithm is stable for the corresponding model. Any number less than 40,000 indicates the program terminated due to the instability in the PML algorithm.

From Table 1 we conclude that the classical PML is unstable if the Poisson's ratio of the model is greater than about 0.38 . Figure 4 also indicates that the relationship between the rate of divergence in the PML and the Poisson's ratio is nonlinear because of the different exponential accumulation speed of the numerical errors. Generally, the higher the Poisson's ratio, the faster the classical PML algorithm diverges. The error accumulates exponentially with the increase of Poisson's ratio. When the Poisson's ratio is greater than 0.4 , none of the simulations can survive more than 8000 loops.

\section{M-PML TECHNIQUE AND ITS STABILITY FOR NEAR-SURFACE EARTH MODELS}

The M-PML technique was developed by Meza-Fajardo and Papageorgiou (2008) to solve the instability problem of classical PML. The basic idea of the M-PML is that the waves simultaneously decay with multiple damping profiles in orthogonal directions. The damping profiles are proportional to each other. For example, in the 2D PML model shown in Figure 1, the damping profile along the $x$ direction can be defined as
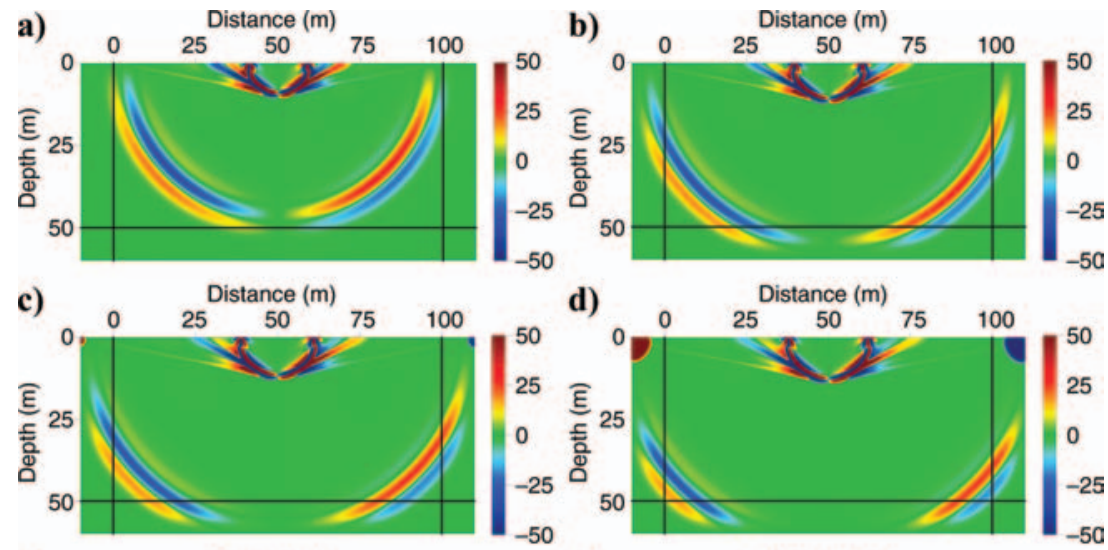

d)
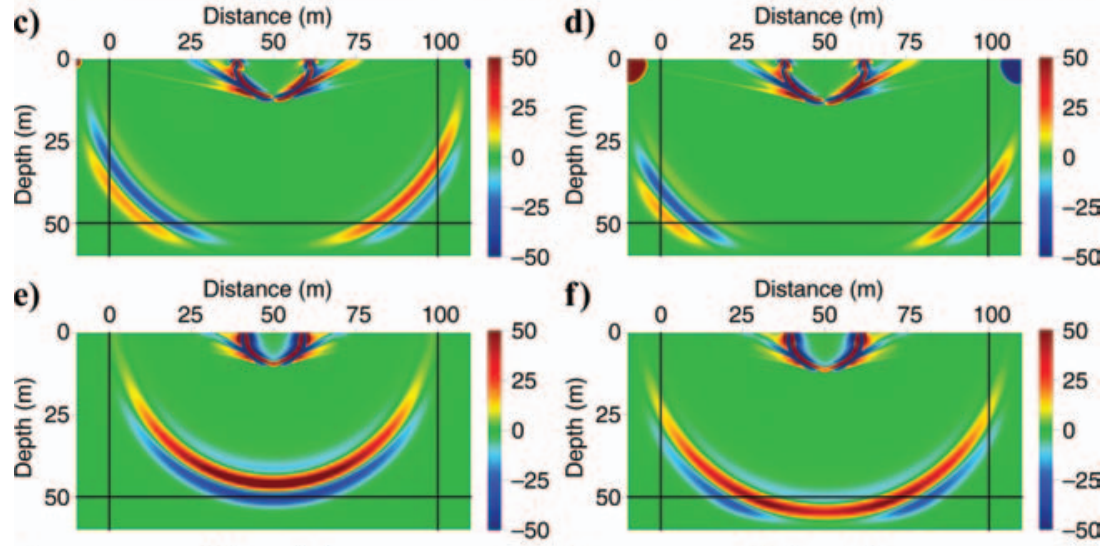

f)
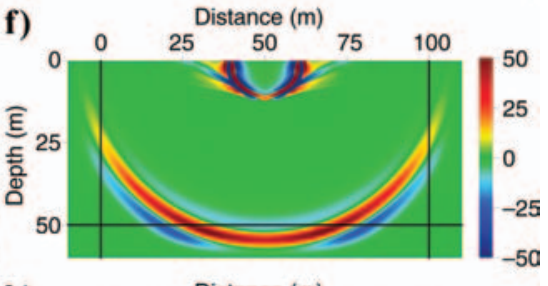

Distance $(\mathrm{m})$

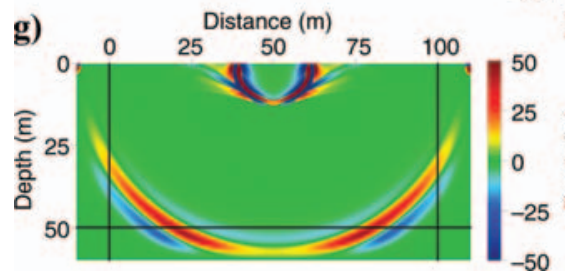

h)

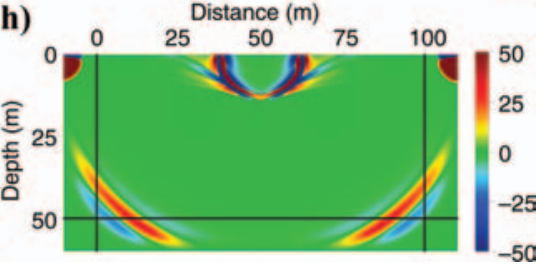

Figure 3. (a) Snapshot of the horizontal $\left(v_{x}\right)$ and vertical $\left(v_{z}\right)$ particle velocities for a high Poisson's ratio earth model with the classical PML $v_{x}$ at $t=115 \mathrm{~ms}$. (b) Snapshot of the horizontal $\left(v_{x}\right)$ and vertical $\left(v_{z}\right)$ particle velocities for a high Poisson's ratio earth model with the classical PML $v_{x}$ at $t=132 \mathrm{~ms}$. (c) Snapshot of the horizontal $\left(v_{x}\right)$ and vertical $\left(v_{z}\right)$ particle velocities for a high Poisson's ratio earth model with the classical PML $v_{x}$ at $t=139 \mathrm{~ms}$. (d) Snapshot of the horizontal $\left(v_{x}\right)$ and vertical $\left(v_{z}\right)$ particle velocities for a high Poisson's ratio earth model with the classical PML $v_{x}$ at $t=149 \mathrm{~ms}$. (e) Snapshot of the horizontal $\left(v_{x}\right)$ and vertical $\left(v_{z}\right)$ particle velocities for a high Poisson's ratio earth model with the classical PML $v_{z}$ at $t=115 \mathrm{~ms}$. (f) Snapshot of the horizontal $\left(v_{x}\right)$ and vertical $\left(v_{z}\right)$ particle velocities for a high Poisson's ratio earth model with the classical PML $v_{z}$ at $t=132 \mathrm{~ms}$. (g) Snapshot of the horizontal $\left(v_{x}\right)$ and vertical $\left(v_{z}\right)$ particle velocities for a high Poisson's ratio earth model with the classical PML $v_{z}$ at $t=139 \mathrm{~ms}$; numerical errors present at the upper-left and upper-right corners. (h) Snapshot of the horizontal $\left(v_{x}\right)$ and vertical $\left(v_{z}\right)$ particle velocities for a high Poisson's ratio earth model with the classical PML $v_{z}$ at $t=149 \mathrm{~ms}$; Numerical errors accumulate to significant values at the upper-left and upper-right corners. 


$$
d_{x}=d_{x}^{x}(x), d_{z}=p^{(z / x)} d_{x}^{x}(x),
$$

where $p^{(z / x)}$ is the proportion coefficient in either the left or right PML. Similarly, the damping profile along the $z$ direction can be defined as

$$
d_{x}=p^{(x / z)} d_{z}^{z}(z), d_{z}=d_{z}^{z}(z),
$$

where $p^{(x / z)}$ is the proportion coefficient in the bottom PML.

Equations 8 and 9 can be considered generalizations of equations 6 and 7 for the classical uniaxial PML. When the proportion coefficient is zero, the multiaxial PML profiles in equations 8 and 9 degrade to the uniaxial profiles. A key characteristic of M-PML is that a single velocity/stress vector is attenuated in multiple directions. While in uniaxial PML, a single vector is always attenuated in only one direction.

Meza-Fajardo and Papageorgiou (2008) suggested the M-PML is stable for an isotropic medium with the existence of surface waves. In their model example, the Poisson's ratio is about 0.24 . For such a model, the instability problem of classical PML only appears if the simulation is performed over the long time duration. It was reported by Festa et al. (2005) that the C-PML technique is more stable than the classical PML for their model. However, in our test the last 10 models listed in

Table 1. Physical parameters of the models for stability tests

\begin{tabular}{|c|c|c|c|}
\hline$\sigma$ & $v_{p} / v_{s}$ & $v_{s}(\mathrm{~m} / \mathrm{s})$ & Termination loop \\
\hline $0.10-0.25$ & $1.50-1.73$ & $347-300$ & 40000 \\
\hline 0.26 & 1.76 & 296 & 40000 \\
\hline 0.27 & 1.78 & 292 & 40000 \\
\hline 0.28 & 1.81 & 287 & 40000 \\
\hline 0.29 & 1.84 & 283 & 40000 \\
\hline 0.30 & 1.87 & 278 & 40000 \\
\hline 0.31 & 1.91 & 273 & 40000 \\
\hline 0.32 & 1.94 & 268 & 40000 \\
\hline 0.33 & 1.99 & 262 & 40000 \\
\hline 0.34 & 2.03 & 256 & 40000 \\
\hline 0.35 & 2.08 & 250 & 40000 \\
\hline 0.36 & 2.14 & 243 & 40000 \\
\hline 0.37 & 2.20 & 236 & 40000 \\
\hline 0.38 & 2.27 & 229 & 40000 \\
\hline 0.39 & 2.35 & 221 & 18702 \\
\hline 0.40 & 2.45 & 212 & 7834 \\
\hline 0.41 & 2.56 & 203 & 5122 \\
\hline 0.42 & 2.69 & 193 & 3863 \\
\hline 0.43 & 2.85 & 182 & 3153 \\
\hline 0.44 & 3.06 & 170 & 2707 \\
\hline 0.45 & 3.32 & 157 & 2400 \\
\hline 0.46 & 3.67 & 142 & 2149 \\
\hline 0.47 & 4.20 & 124 & 1941 \\
\hline 0.48 & 5.10 & 102 & 1772 \\
\hline 0.49 & 7.14 & 73 & 1653 \\
\hline
\end{tabular}
of classical PML.
Table 1 whose Poisson's ratios are greater than 0.39 diverge quickly for the classical PML and C-PML algorithm.

Models listed in Table 1 are used again for the numerical tests designed to check the stability of the M-PML algorithm for near-surface earth models with high Poisson's ratios. All model parameters are exactly the same as those used in the previous analysis of classical PML. The only difference is the use of the multiaxial technique. During implementation, the proportion coefficients $p^{(z / x)}$ and $p^{(x / z)}$ were set to 1.0. No violation of the velocity threshold was observed during the modeling tests. The M-PML algorithm is convergent and stable for all models with Poisson's ratios that vary from 0.10 to 0.49 .

To demonstrate the stability of the M-PML technique and its absorbing effectiveness, we apply the M-PML technique to the homogeneous half-space model (Figure 3) where the classical uniaxial PML is unstable. Figure 5 presents the wavefield snapshots of vertical particle velocities at the same time instants as shown in Figure 3. Prior to the wavefronts reaching the external model edges (Figure 5a and 5b), the M-PMLs appear similar to the classical uniaxial PMLs. For the $t=139 \mathrm{~ms}$ (Figure 5c) and $t=149 \mathrm{~ms}$ (Figure 5d), no significant numerical error appears in the snapshots for the M-PML technique. The simulation completed successfully without violating the thresholds detailed for previous numerical tests.

\section{THE SIMPLIFIED M-PML AND ITS APPLICATION}

It is noteworthy that the only numerical errors appear in the upper part of the left and right PMLs near the free surface in the wavefield snapshots in Figure 3. In the bottom PML where only body waves exist, the classical PML works efficiently with no significant accumulative errors. A range of numerical tests (detailed results not shown here) run on the models with various Poisson's ratios result in similar observations. The snapshots from the tests suggest the initial significant numerical error always comes from the upper-left and upper-right corner of the PMLs (for the 2D case) due to the existence of the free surface.

Figure 6a displays the wavefield snapshots for a model using the classical PML without a free surface. The model is a

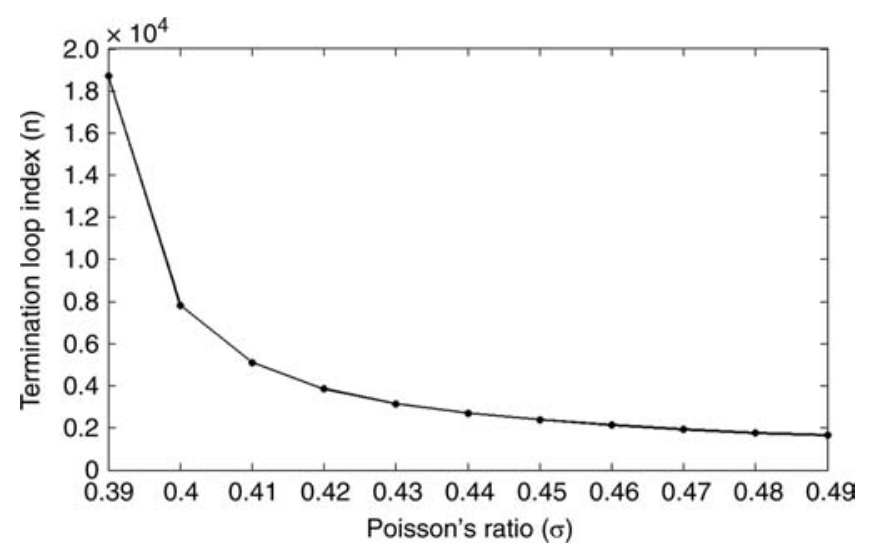

Figure 4. A nonlinear relation between the divergence speed of the classical PML and the values of Poisson's ratios, where $n$ is the loop index when the program terminates due to the violation of velocity threshold, and $\sigma$ is the Poisson's ratio. The dots are the computed $(\sigma, n)$ values extracted from Table 1 when the classical PML is unstable. 
$100 \times 100 \mathrm{~m}$ homogeneous unbounded medium. Four classical PMLs are attached at each edge of the model. The source is located at the center $(x=50 \mathrm{~m}, z=50 \mathrm{~m})$ of the model. The physical parameters $\left(v_{p}, v_{s}\right.$, and $\left.\rho\right)$ are exactly the same as those used for the model in Figure 3. The classical PML is unstable when the free surface exists in this high Poisson's ratio medium. However, when there is no free surface, the only seismic waves in the medium are the body waves (P-waves and S-waves). In Figure $6 \mathrm{~b}$, the P-wave and S-wave are efficiently absorbed by the PMLs with neither spurious reflections nor significant accumulative errors. The classical PML is stable without the existence of the free surface even when the Poisson's ratio is high. This is consistent with the claim that the instability of the classical uniaxial PML for the earth models with high Poisson's ratios is due to the existence of the free surface. Specifically, the instability of the classical PML is mainly influenced by the complex wave phenomena related to the free surface.

The amplitude of Rayleigh waves decays exponentially with increasing depth. For a model with a large vertical dimension, the energy of Rayleigh waves near the bottom edge is usually weak enough to be negligible. In this case, the multiaxial technique for the bottom PML is unnecessary because only body waves are involved. Moreover, the algorithm is stable for the left and right absorbing strips after only applying the multiaxial technique to the upper part of the PMLs. Hence, the M-PML can be simplified so that only the upper-left and upperright corners need multiple damping profiles. For other parts of the PML strips, only one damping profile is used consistent with the classical uniaxial PML technique. This can reduce the memory cost for storing M-PML profiles during program implementation. It also has the potential to save CPU time for large scale modeling because there is no need to compute the terms with multiple PML damping coefficients outside the upper-left and upper-right corners.

Waves in the M-PMLs are attenuated exponentially in $x$ - and $z$-directions due to the introduction of the proportional damping profiles. For Rayleigh waves whose amplitudes already decrease exponentially with increasing of depth, the energy reduces much faster than that of body waves in the vertical direction. Modeling tests show that a satisfactory absorbing effectiveness can be archived in most cases by setting the vertical thickness of the upper M-PML zone to a half of the dominant wavelength of the P-waves near the free surface.

In theory, the horizontal interface between the upper M-PML zone and beneath the uniaxial PML zone in the simplified M-PML method will generate spurious reflections due to the abrupt change of absorbing parameters in the vertical direction. The spurious reflections could propagate as multiples to the free surface and contaminate the synthetic wavefield. However, these spurious reflections are negligible in practice when modeling Rayleigh waves in near surface materials if the thickness of the upper MPML zone is set appropriately. This is because the energy of the Rayleigh waves at the interface between the M-PML and the uniaxial PML is already attenuated to be weak enough comparing to its original value on the free surface. The spurious reflections from the body waves are also insignificant because their maximum amplitudes after attenuation are usually less than $1 \%$
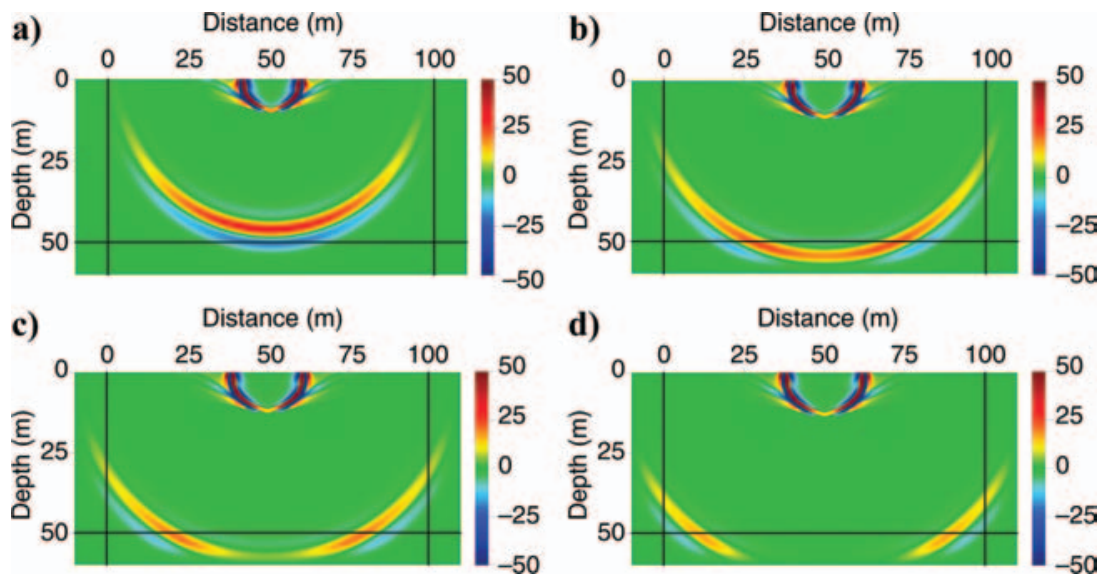

Figure 5. (a) Snapshot of the vertical particle velocities for the exactly same model used in Figure 3 but with the M-PML applied. The time instant is $t=115 \mathrm{~ms}$. No significant numerical errors are observed. The simulation was also completed with no violation to the velocity threshold. (b) Snapshot of the vertical particle velocities for the exactly same model used in Figure 3 but with the M-PML applied. The time instant is $t=132 \mathrm{~ms}$. No significant numerical errors are observed. The simulation was also completed with no violation to the velocity threshold. (c) Snapshot of the vertical particle velocities for the exactly same model used in Figure 3 but with the M-PML applied. The time instant is $t=139 \mathrm{~ms}$. No significant numerical errors are observed. The simulation was also completed with no violation to the velocity threshold. (d) Snapshot of the vertical particle velocities for the exactly same model used in Figure 3 but with the M-PML applied. The time instant is $t=149 \mathrm{~ms}$. No significant numerical errors are observed. The simulation was also completed with no violation to the velocity threshold.
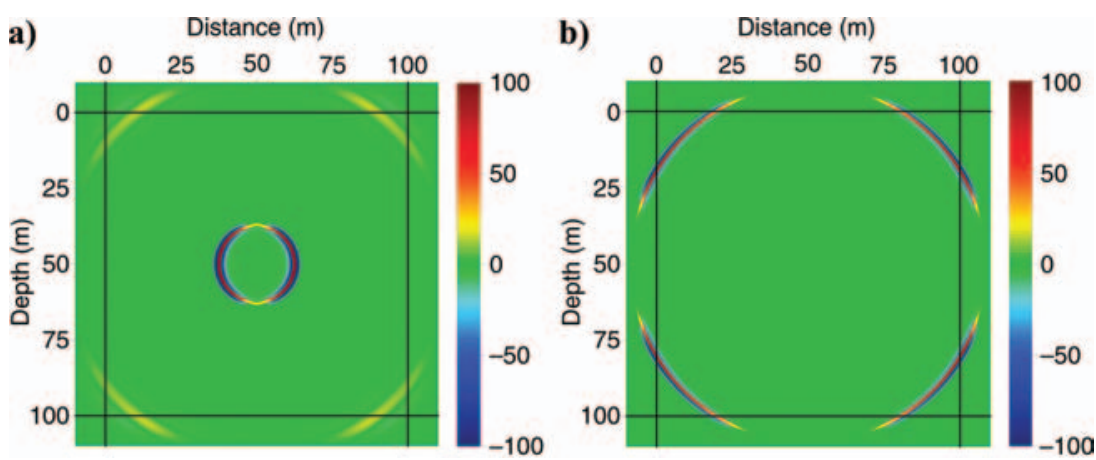

Figure 6. (a) Snapshot of the vertical particle velocity for an unbounded homogeneous earth model with classical PML. The Poisson's ratio of the medium is 0.48 . The source is located at the center of the model. Snapshot at $t=149 \mathrm{~ms}$, when the P-wave enters the PMLs. Figure $6 \mathrm{a}$ and $6 \mathrm{~b}$ illustrate that the classical PML is stable without the existence of the free surface even when the Poisson's ratio is high. (b) Snapshot of the vertical particle velocity for an unbounded homogeneous earth model with classical PML. The Poisson s ratio of the medium is 0.48 . The source is located at the center of the model. Snapshot at $t=600 \mathrm{~ms}$, when the S-wave enters the PMLs. Figure 6a and $6 \mathrm{~b}$ illustrate that the classical PML is stable without the existence of the free surface even when the Poisson s ratio is high. 
of the peak amplitude of the Rayleigh waves in the high Poisson's ratio earth models.

The simplified M-PML is stable through the numerical tests with all the models listed in Table 1. Furthermore, we find through numerical modeling that a constant proportion coefficient $p^{(z / x)}=p^{(x / z)}=1.0$ can make the M-PML stable for all the models regardless of Poisson's ratios. The values used in MezaFajardo and Papageorgiou's (2008) tests (0.1 and 0.15) for isotropic media; however, cause instability of M-PML for our cases.

For heterogeneous earth models, the simplified M-PML is still stable and efficient. A two layered earth model (Xia et al., 2007) is used to demonstrate the application of the simplified M-PML to a heterogeneous medium. The model's physical parameters are listed in Table 2. The dispersion image extracted from the synthetic record, which indicates the relationship of Rayleigh-wave phase velocities and the frequencies, can be used to verify the accuracy of the simulation. If the synthetic record is not contaminated by spurious reflections, the energy concentration on the dispersion image should match the theoretical dispersion curves. Figure 7 is the synthetic shot gather for the model generated by FD modeling with the simplified M-PML technique. The source is a first derivative of the Gaussian function with dominant frequency $f=20 \mathrm{~Hz}$ and time zero delay $t_{0}=60 \mathrm{~ms}$. The trace interval and the nearest offset are $1 \mathrm{~m}$. The proportion coefficients for the PML damping profiles in $x$ and $z$ directions are 1.0. There are no significant spurious reflections observed on the shot gather. The dispersion image (Figure

Table 2. Physical parameters of a layered earth model (Xia et al., 2007).

\begin{tabular}{lccccc} 
Layer & Thickness $(\mathrm{m})$ & $v_{p}(\mathrm{~m} / \mathrm{s})$ & $v_{s}(\mathrm{~m} / \mathrm{s})$ & $\rho\left(\mathrm{kg} / \mathrm{m}^{3}\right)$ & $\sigma$ \\
\hline 1 & 10 & 800 & 200 & 2000 & 0.47 \\
2 & $\infty$ (half-space) & 1200 & 400 & 2000 & 0.44 \\
\hline
\end{tabular}

8) generated by the high resolution linear Radon transform (Luo et al., 2008) agrees well with the theoretical dispersion curves (Schwab and Knopoff, 1972), which indicates the Rayleighwave information is accurately modeled without contamination from spurious reflections or numerical errors.

\section{DISCUSSION}

The snapshots in Figure 3 indicate that the numerical errors always arise from the corner of the free surface and the truncated edges of the model. In Figure 3c, significant error appears immediately after the wavefronts of the P-S (compressional wave to shear wave) converted waves on the free surface touched the truncated boundary. For the tests in this paper, the physical truncation on the model edge is implemented by the Dirichlet boundary conditions. However, the tests without the Dirichlet boundary conditions also yield the instability. Another simulation with a vertical free surface didn't survive either. The detailed generation mechanism of these numerical errors needs sophisticated mathematical error analysis. However, it can be concluded that the instability is a combination effect of the free surface condition on the top and the physical truncation on the left and right edges in a high Poisson's ratio earth model.

To test the stability of the classical PML incorporating with internal interface where high Poisson's ratio appears, we performed a modeling for a two-layered earth model, whose top layer is a Poisson's solid $(\sigma=0.25)$ and bottom layer has a high Poisson's ratio of 0.48 . The simulation completed without any instability observed. This suggests that the instability of the uniaxial PML is controlled by the high Poisson's ratio materials near the free surface.

Mesa-Fajardo and Papageorgiou (2008) point out that the M-PML proportion coefficients need to take higher values to stabilize the medium when the damping profiles grow fast. When small damping ratios are used, the M-PML has to be thick enough to yield stable absorptions. In near-surface modeling that focuses on Rayleigh waves, the absorbing boundary
Figure 7. Synthetic shot gather for a two-layer earth model (Xia et al., 2007) using the simplified M-PML technique. Rayleigh waves are dispersive due to the heterogeneity of the medium. There are no significant spurious reflections on the shot gather.

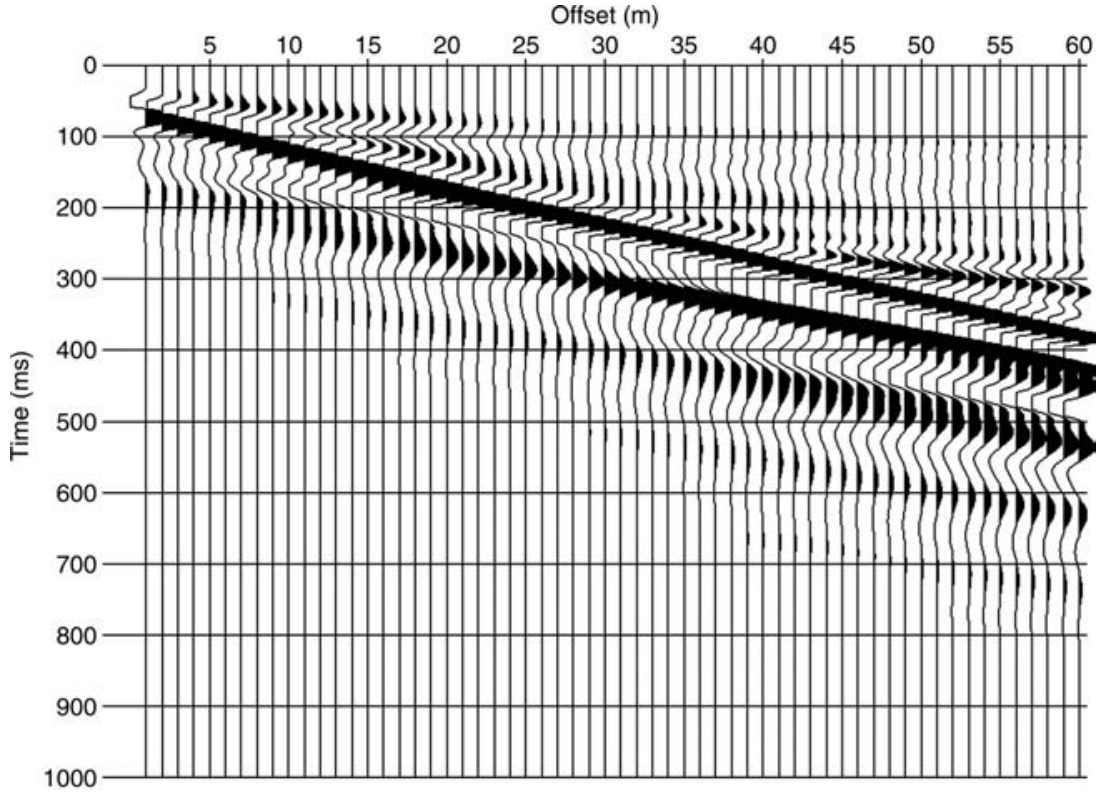




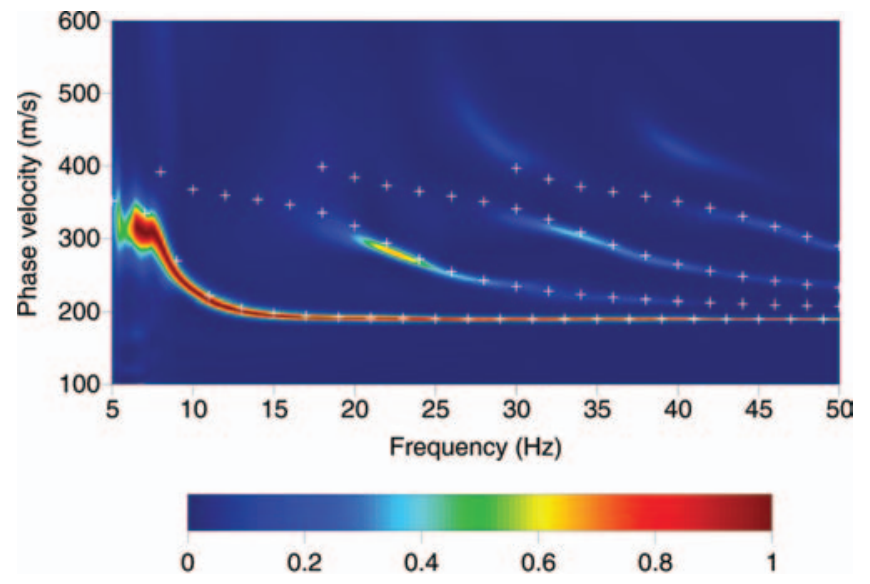

Figure 8. Dispersion image computed from the synthetic shot gather of the two-layer earth model. The color scale of the image represents the distribution of the normalized wavefield energy in frequency-velocity domain. The crosses represent the theoretical phase velocities calculated by the Knopoff method (Schwab and Knopoff, 1972). The energy concentration on the dispersion image agrees well with the theoretical dispersion curves, which indicates Rayleigh waves are modeled accurately without contamination by spurious reflections or numerical errors.

layers are usually designed to be as thin as possible to reduce the computational cost due to the employment of small grid spacing. This impels us to use relatively greater values (e.g., 1.0) than those used in Mesa-Fajardo and Papageorgiou's examples $(0.1$ and 0.15$)$. However, high values of the proportion coefficients increase the spurious reflections due to the reflection coefficients of PML being nonzero after discretization. The value of 1.0 for the proportion coefficients used in this paper is a compromised solution that can stabilize the M-PML with acceptable absorbing effectiveness for the most near-surface earth models. Optimum values of the proportion coefficients may differ from the proposed value depending on the specific model settings.

\section{CONCLUSIONS}

The classical uniaxial PML technique is unstable for nearsurface earth models when the Poisson's ratio is high (greater than 0.38 in our test examples). The higher the Poisson's ratio, the faster the classical PML algorithm diverges. The existence of the free surface is the reason for this instability. The free-surface related complex wave phenomena play important roles in the fast accumulation of numerical errors inside the PMLs. Numerical tests on the models with Poisson's ratios vary from 0.10 to 0.49 and demonstrate that the M-PML technique is stable if the proportion coefficient of the PML damping profiles is set appropriately. For 2D seismic modeling focusing on Rayleigh waves, the multiaxial technique is only necessary for the free space (upper-left and upper-right) corners of the PML. For the other grids inside the PMLs, the conventional uniaxial PML is stable enough to absorb the spurious reflections. Numerical tests show that the proportion coefficients of the multiaxial PML damping profiles in $x$ - and $z$-directions can be set to a constant of 1.0. For isotropic elastodynamics, this constant proportion coefficient is sufficient to make the M-PML algorithm stable for all models regardless of
Poisson's ratio. The M-PML can be simplified without losing its stability by implementing the multiaxial technique only to the upper corners of the PMLs near the free surface. For homogeneous and heterogeneous earth models with high Poisson's ratios, Rayleigh waves can be simulated accurately through the application of this simplified M-PML technique. All the analysis in this paper is based on 2D FD modeling in the time domain; however, extension to the 3D domain is straightforward.

\section{ACKNOWLEDGMENTS}

We greatly appreciate the comments and suggestions from associate editor Jeff Shragge, Sjoerd de Ridder, Aaron Girard, and other two anonymous reviewers that significantly improved the quality of the manuscript.

\section{REFERENCES}

Aki, K., and P. G. Richards, 2002, Quantitative seismology, second edition: University Science Books

Bécache, E., S. Fauqueux, and P. Joly, 2003, Stability of perfectly matched layers, group velocities and anisotropic waves: Journal of Computational Physics, 188, no. 2, 399-433, doi:10.1016/S00219991(03)00184-0.

Bérenger, J., 1994, A perfectly matched layer for the absorption of electromagnetic waves: Journal of Computational Physics, 114, no. 2, 185200, doi:10.1006/jcph.1994.1159.

Calderón-Macías, C., and B. Luke, 2007, Improved parameterization to invert Rayleigh-wave data for shallow profiles containing stiff inclusions: Geophysics, 72, no. 1, U1-U10.

Carcione, J. M., G. C. Herman, and A. P. E. ten Kroode, 2002, Seismic modeling: Geophysics, 67, 1304-1325.

Cerjan, C., D. Kosloff, R. Kosloff, and M. Reshef, 1985, A nonreflecting boundary condition for discrete acoustic and elastic wave equations: Geophysics, 50, 705-708.

Chew, W., and Q. H. Liu, 1996, Perfectly matched layers for elastodynamics: a new absorbing boundary condition: Journal of Computational Acoustics, 4, 341-359.

Collino, F., and C. Tsogka, 2001, Application of the PML absorbing layer model to the linear elastodynamic problem in anisotropic heterogeneous media: Geophysics, 66, 294-307.

Festa, G., E. Delavaud, and J. P. Vilotte, 2005, Interaction between surface waves and absorbing boundaries for wave propagation in geological basins: 2D numerical simulations: Geophysical Research Letters, 32, L20306.

Festa, G., and S. Nielsen, 2003, PML absorbing boundaries: Bulletin of Seismological Society of America, 93, 891-903.

Graves, R. W., 1996, Simulating seismic wave propagation in 3D elastic media using staggered-grid finite differences: Bulletin of Seismological Society of America, 86, 1091-1106.

Komatitsch, D., and R. Martin, 2007, An unsplit convolutional perfectly matched layer improved at grazing incidence for the seismic wave equation: Geophysics, 72, no. 5, SM155-SM167.

Kristek, J., P. Moczo, and R. J. Archuleta, 2002, Efficient methods to simulate planar free surface in the 3D 4th-order staggered-grid finite-difference schemes: Studia Geophysica et Geodaetica, 46, 355-381.

Levander, A. R., 1988, Fourth-order finite-difference P-SV seismograms: Geophysics, 53, 1425-1436.

Luo, Y., J. Xia, R. D. Miller, Y. Xu, J. Liu, and Q. Liu, 2008, Rayleigh-wave dispersive energy imaging using a high-resolution linear Radon transform: Pure and Applied Geophysics, 165, 903922 .

Madariaga, R., 1976, Dynamics of an expanding circular fault: Bulletin of Seismological Society of America, 66, 639-666.

Meza-Fajardo, K. C., and A. S. Papageorgiou, 2008, A nonconvolutional, split-field, perfectly matched layer for wave propagation in isotropic and anisotropic elastic media: Stability analysis: Bulletin of Seismological Society of America, 98, 1811-1836.

Mittet, R., 2002, Free-surface boundary conditions for elastic staggeredgrid modeling schemes: Geophysics, 67, 1616-1623.

Moczo, P., J. Kristek, V. Vavryčuk, R. J. Archuleta, and L. Halada, 2002, $3 \mathrm{D}$ heterogeneous staggered-grid finite-difference modeling of seismic motion with volume harmonic and arithmetic averaging of elastic moduli and densities: Bulletin of Seismological Society of America, 92 3042-3066. 
Nazarian, S., and K. H. Stokoe II, 1984, In situ shear wave velocities from spectral analysis of surface waves: 8th Conference on Earthquake Engineering, 3, 31-39.

Saenger, E. H., and T. Bohlen, 2004, Finite-difference modeling of viscoelastic and anisotropic wave propagation using the rotated staggeredgrid: Geophysics, 69, 583-591.

Schwab, F. A., and L. Knopoff, 1972, Fast surface wave and free mode computations, in B. A. Bolt, ed., Methods in computational physics: Academic Press, 87-180.

Socco, L. V., S. Foti, and D. Boiero, 2010, Surface-wave analysis for building near-surface velocity models — Established approaches and new perspectives: Geophysics, 75, no. 5, 75A83-75A102.

Virieux, J., 1986, P-SV wave propagation in heterogeneous media: Velocity-stress finite-difference method: Geophysics, 51, 889-901.

Xia, J., R. D. Miller, and C. B. Park, 1999, Estimation of near-surface shearwave velocity by inversion of Rayleigh waves: Geophysics, 64, 691-700.
Xia, J., R. D. Miller, C. B. Park, J. A. Hunter, J. B. Harris, and J. Ivanov, 2002, Comparing shear-wave velocity profiles inverted from multichannel surface wave with borehole measurements: Soil Dynamics and Earthquake Engineering, 22, 181-190.

Xia, J., R. D. Miller, C. B. Park, and G. Tian, 2003, Inversion of high frequency surface waves with fundamental and higher modes: Journal of Applied Geophysics, 52, 45-57.

Xia, J., Y. Xu, and R. D. Miller, 2007, Generating image of dispersive energy by frequency decomposition and slant stacking: Pure and Applied Geophysics, 164, 941-956.

Xia, J., Y. Xu, R. D. Miller, and C. Chen, 2006, Estimation of elastic moduli in a compressible Gibson half-space by inverting Rayleigh wave phase velocity: Surveys in Geophysics, 27, 1-17.

Xu, Y., J. Xia, and R. D. Miller, 2007, Numerical investigation of implementation of air-earth boundary by acoustic-elastic boundary approach: Geophysics, 72, no. 5, SM147-SM153. 